\title{
An in situ technique for the assessment of adhesive properties of a joint under load
}

\author{
M. K. Budzik · J. Jumel • M. E. R. Shanahan
}

Received: 16 June 2011 / Accepted: 13 September 2011 / Published online: 18 October 2011

(C) The Author(s) 2011. This article is published with open access at Springerlink.com

\begin{abstract}
Slow crack propagation in adhesive bonded joints has been characterised using an asymmetric wedge test. Crack position was evaluated from strain gauge measurements, both in the debonded part of the joint and in the bonded zone. Test temperature was changed during loading, giving insight into bond evolution. The technique allows accurate, and virtually continuous, determination of crack position to be made, and therefore the evaluation of crack speed versus fracture energy curves, as well as elastic properties of the adhesive layer. This technique also enables the monitoring of crack propagation in controlled environmental conditions to be performed, without interruption of exposure for measurements. By using a Winkler elastic foundation model to analyse results, the method seems to be the first to describe a process zone, or region where the adhesive is significantly strained under load, and a finite length specimen effect, manifested by crack front acceleration during the final stage of the test. The method was found to offer great potential to study in situ fracture and bulk adhesive properties.
\end{abstract}

M. K. Budzik ( $\varangle)$ · J. Jumel · M. E. R. Shanahan Institute de Mécanique et d'Ingenierie, Université de Bordeaux, CNRS UMR 5469, Bâtiment A4, 351 Cours de la Libération, 33405 Talence Cedex, France e-mail: michal.k.budzik@gmail.com

M. K. Budzik

Material Engineering and Welding Department, University of Technology Gdansk, 80233 Gdansk, Poland
Keywords Process zone - Adhesive bonding . Crack propagation $\cdot$ Wedge test

\section{Introduction}

The wedge test has become a popular method for assessment of the durability of adhesively bonded joints (Bardis and Kedward 2001; Bujanda et al. 2008; Adams et al. 2009). This inexpensive and reliable method has been standardised (ASTM D3762) and is frequently used to determine optimal bonding conditions (adherend, adhesive, cure cycle, surface preparation, etc.) and environmental resistance (Blackman and Kinloch 2001).

The basic test consists of two (thin) planar adherends bonded together. A 'wedge' is inserted into an unbonded end to produce double cantilever beam geometry, leading to loading at constant imposed displacement. Elastic (strain) energy in the adherends drives crack initiation and propagation along the bondline. Energy released rate decreases as $a^{-4}$, where $a$ is crack length, making stable crack propagation possible. The crack propagation is often observed directly using optical microscopy (Aglan and Abdo 1996; Sargent 2005; Adams et al. 2009), generally leading to discontinuous measurements. The principal, quantitative datum is crack position from which energy release rate is calculated. Other approaches have also been reported (Crosley and Ripling 1991; Sener et al. 2002; Nayeb-Hashemi et al. 2004; Hwang et al. 2005; Pardoen et al. 2005). 
Today, the development of composite materials and other lightweight structures is accompanied by an increasing use of adhesive of structural joining (Baker 1999; Baldan 2004). This necessitates reliability in design, and consequently better prediction of damage and failure time. Up to now, the wedge test has been regarded as a safe lifetime prediction technique (Boller et al. 2001). To take advantage of the simple experimental arrangement and low cost of the wedge test, but in order to monitor crack propagation more quantitatively, we developed an instrumented asymmetric wedge test (one adherend much more rigid than the other Budzik et al. 2009a,b, 2011a). In this technique, strain gauges are bonded on the outer skin of the flexible adherend loaded in simple bending (sometimes referred to as the backface technique Crocombe et al. 2002; Hadavinia et al. 2002). Surface strain variation is linear along the free region (e.g. Budzik et al. 2011a), with the maximum at the crack front and zero at the wedge position (no bending moment). Apparent crack length evolution is determined by monitoring the slope of strain variation along the beam with time. Continuous energy release rate, $G$ (and therefore fracture energy, $G_{\mathrm{c}}$ ), versus crack speed, $(d a / d t)$, data can be obtained.

The simplest analysis considers rigid clamping of the flexible adherend the bonded zone (Budzik et al. 2009a,b). In the case of a 'soft' adhesive joint (thick bondline, soft adhesive, or short crack), the overall sample compliance is higher than expected from Euler-Bernoulli beam bending theory. The 'root rotation' effect, quantitatively taken into account with Winkler (Winkler 1867; Kanninen 1973) or Pasternak (Ma et al. 2009) elastic foundation models, can be observed with strain gauge or interferometric techniques (Budzik et al. 2011a,b). We have recently shown that in stationary conditions (no crack growth), extension of the adhesive near the crack tip normal to the bondline (referred to as the 'process zone') is directly related to the tensile elastic properties of the adhesive layer and can be measured. It should be emphasised that the process zone as described in the following is a phenomenological description of the region in which the adhesive is significantly strained prior to fracture, and not directly related to the known fracture process zone-FPZ (Shah 1990; Amrutharaj et al. 1995). This nomenclature was chosen deliberately to emphasise the physical similarity of the process zone, as a constrained zone preceding the crack.

In the present contribution we show the instrumented wedge test to be a reliable, quantitative, yet still stand alone, and inexpensive technique to characterise the behaviour of adhesively bonded joints. After temperature change (during the test), both crack growth and strain properties of the bondline evolve. Finally, we present analysis of the test geometry with finite length specimen effects.

\section{Experimental}

The wedge test used was asymmetric (Budzik et al. 2009a), applied to dissimilar aluminium plates (see Fig. 1). The thin or flexible adherend was an aluminium copper alloy, AW2024-T3 (Alcoa, Pittsburgh, PA, USA), of thickness $h=1.6 \mathrm{~mm}$. Young's modulus $E$ and Poisson's ratio $v$, as obtained by ultrasound TTM (through transmission method) were $70 \pm 2 \mathrm{GPa}$ and 0.3. The rigid, or thick, plate was of aluminium magnesium alloy AW5754 (Alcoa, Pittsburgh, PA, USA)

Fig. 1 Experimental arrangement of the wedge test

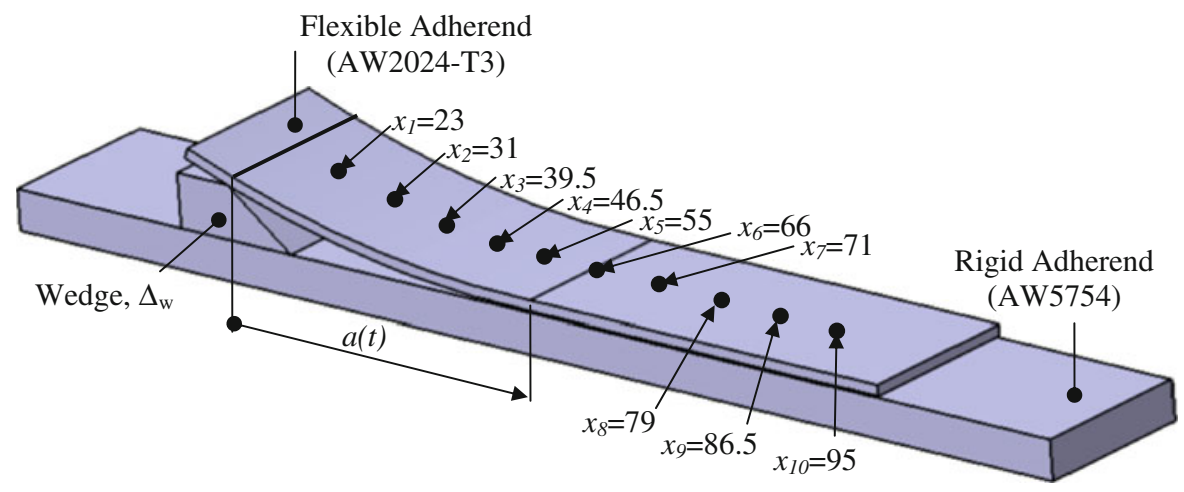


with a thickness of $H=6 \mathrm{~mm}$. Properties of the rigid substrate material, as given by the supplier, are Young's modulus $=67 \mathrm{GPa}$ and Poisson ratio $=0.3$. The relative flexural rigidity (ratio of the cubes of thickness multiplied by the appropriate Young's modulus), is ca. 52, so the terms rigid and flexible are reasonable.

Prior to bonding, aluminium surfaces were polished to 2,400 grid emery paper and sand blasted with Salox $\mathrm{Al}_{2} \mathrm{O}_{3} 105 \mu \mathrm{m}$ particles. Surfaces were washed in ethanol using an ultrasonic cleaner (Tovatech LLC, South Orange, New Jersey, USA) for $10 \mathrm{~min}$ at $35 \mathrm{kHz}$ and at $25^{\circ} \mathrm{C}$. Subsequently, the flexible adherend of length, $l=120 \mathrm{~mm}$ was bonded to the rigid member of length $L=180 \mathrm{~mm}$, leaving a free zone, or initial crack length, $a=35 \mathrm{~mm}$. The initial length of bonded zone was $l_{\text {adh }}=70 \mathrm{~mm}$ while width $b$ of the entire system was $25 \mathrm{~mm}$.

The plates were bonded using a commercial epoxy resin [Araldite Cristal (Bostik, La Défense, Paris, France)] consisting of Di-Glycidyl Ether of Bisphenol A (DGEBA) resin of average molecular weight $<700$ cured with an amine agent: $\mathrm{N}(3$ dimethylaminopropyl)-1,3 propylenediamine. Crosslinking was effected at ambient temperature $\left(\mathrm{ca} .23^{\circ} \mathrm{C}\right)$ for a week under 0.3 bar pressure and at $c a$. 55\% RH. Two PTFE strips were used to ensure sharp bonded zone edges as well as a bondline thickness $e=0.25 \mathrm{~mm}$, homogenous along the bonded zone as measured with a digital micro camera (Dino-Lite Pro-IS Production S.A.01633 St. Genis Pouilly, France).

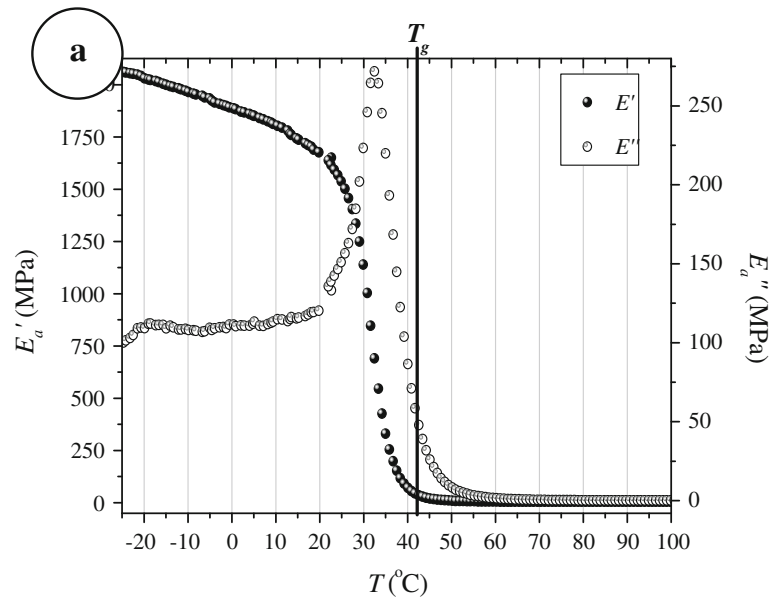

\subsection{Characterisation of the adhesive}

Mechanical properties of the adhesive were estimated using dynamic mechanical thermal analysis (DMTA). An Areva MetraVib DMA+150 (Limonest, France) analyser was used to study tensile properties of the Araldite Cristal adhesive. Crosslinking of the adhesive sample was conducted as above, while geometry was $10 \times 5 \times 1 \mathrm{~mm}$ (length $\times$ width $\times$ thickness). Excitation frequency was $1 \mathrm{~Hz}$ with a constant amplitude of $40 \mu \mathrm{m}$, while the temperature, $T$, range was -25 to $100^{\circ} \mathrm{C}$. The properties estimated were storage modulus, $E^{\prime}$, loss modulus $E^{\prime \prime}$, Young's modulus, $E_{A}=\sqrt{E^{\prime 2}+E^{\prime \prime 2}} \approx E^{\prime}$, and dissipation factor (or loss tangent) $\tan \delta=E^{\prime \prime} / E^{\prime}$. Results of the DMTA analysis are presented in Fig. 2.

The adhesive has a glass transition at $c a \cdot 40^{\circ} \mathrm{C}$ $\left(42.5^{\circ} \mathrm{C}\right)$, as indicated by the maximum of the loss tangent. Between 30 and $40^{\circ} \mathrm{C}$, Young's modulus decreases by a factor of $c a$. 10 .

\subsection{Instrumented wedge test in elevated temperature}

The instrumented wedge test is based on (time-dependent) strain recorded on the outer surface of the flexible adherend (Crosley and Ripling 1991). After preparation, ten strain gauges (Vishay Micro-Measurements, EA-13-060LZ-120/E, of nominal resistance $120 \Omega$, Vishay, Malvern, PA, USA) were attached to the outer surface of the flexible adherend along the longitudinal

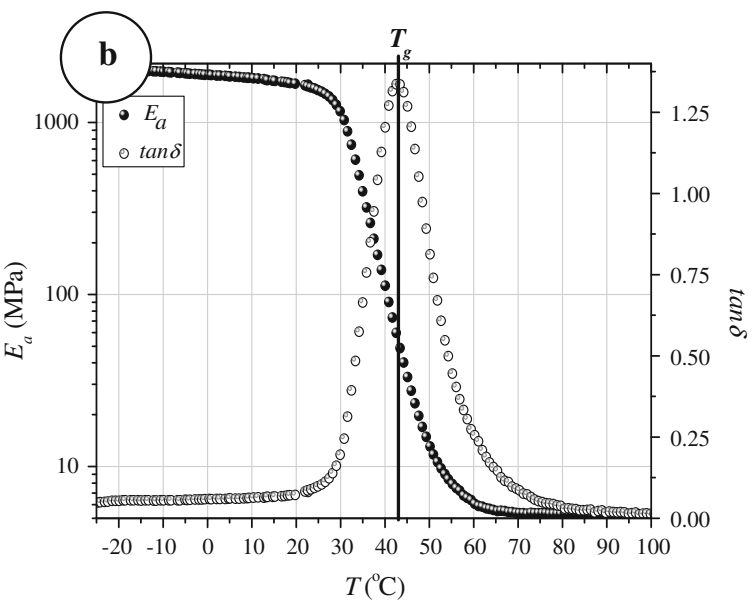

Fig. 2 Results of DMTA at a frequency of $1 \mathrm{~Hz}$. $\mathbf{a} E^{\prime}$ and $E^{\prime \prime}$ and $\mathbf{b} E$ and $\tan \delta$ versus temperature 
axis (see Fig. 1). The sample was pre-cracked with a $5 \mathrm{~mm}$ thick wedge (to produce a sharp, characteristic crack tip), giving a nominal crack length, $a$, of $c a$. $65 \mathrm{~mm}$. Thus, six gauges being initially in the free (unbonded) zone and four in the bonded part of the joint.

The wedge test was initiated at $27^{\circ} \mathrm{C}$ and at a relative humidity of $60 \%$ in a Memmert D 06061 Model 500 oven cabin (Memmert GmbH + Co. KG, Schwabach, Germany), by inserting a wedge between the plates. The asymmetric, aluminium wedge, of thickness $\Delta_{w}=6.8 \mathrm{~mm}$ (thus, the effective mouth opening distance is given by: $\Delta=\Delta_{w}-e$ ), was inserted whilst strain gauge recordings were made using a Wheatstone bridge arrangement (Vishay Micromeasures 2100 System Multi Channel Signal Conditioner/Amplifier with ten modules of Model 2120 B and one module of Model 2110 B Power Supply). The crack was left to propagate at its self-determined rate until this decreased to ca. $0.01 \mathrm{mmh}^{-1}$. The temperature was then increased to $40^{\circ} \mathrm{C}$ at $0.5^{\circ} \mathrm{C} / \mathrm{min}$.

\section{Theory}

In the instrumented wedge test, the flexible adherend effectively becomes a load cell, its curvature being measured by surface strain gauges. As previously shown, limiting the gauges to the free part of the joint only, and assuming encastré cantilever beam boundary conditions with Euler-Bernoulli (EB) formalism, an estimate of crack length may be made. However, the result overestimates the real value (Budzik et al. 2011a), but more importantly, useful information from the process zone is lost. The rudiments are presented below.

\subsection{Simple Euler-Bernoulli (EB) beam}

From the Euler-Bernoulli (EB) beam equation ( $E I$ $d^{2} z / d x^{2}=M=F x$, where $E$ is beam Young's modulus, $I$ its 2 nd moment of area, $z$ the beam displacement with respect to its unloaded position (deflection), $M$ bending moment, $F$ force exerted by the wedge and $x$ distance along the undeformed beam from the origin at wedge contact) we obtain:

$$
\begin{aligned}
z(x) & =\frac{F}{E I}\left(\frac{1}{6} x^{3}-\frac{1}{2} a^{2} x+\frac{1}{3} a^{3}\right) \\
& =\frac{\Delta}{2}\left[\left(\frac{x}{a}\right)^{3}-3\left(\frac{x}{x}\right)+2\right],
\end{aligned}
$$

where $a$ is separation, or crack length. For a rectangular section, $I=b h^{3} / 12$, where $b$ and $h$ are width and thickness. In Eq. (1), no allowance is made for shear in the $z$ direction due to $F$ (Crosley and Ripling 1991), but given the inherent flexibility due to a low ratio of $h / a$, this is considered to be reasonably negligible. Standard boundary conditions of $z(a)=d z / d x(a)=0$ and $z(0)=\Delta$ (wedge thickness at contact) are used, neglecting effects of elastic foundation (Williams 1989; Cotterell et al. 2006). (In this treatment, transverse shear may be neglected since the ratio $h / a$ is small.)

The second differential of $z(x)$ is, to a good approximation, equal to local adherend curvature at $x$, i.e. $R^{-1}(x)$. Assuming the adherend to be isotropic and homogeneous, and of constant thickness, $h$, its strain on the outer (i.e. unbonded) surface, $\varepsilon_{s}=\varepsilon h / 2$ ) (generally negative), is directly related to local curvature and can be given by:

$\left|\varepsilon_{s}(x)\right|=\frac{3 \Delta h x}{2 a^{3}}$.

The linear relationship (2) between $\left|\varepsilon_{S}(x)\right|$ and $x$, for a given crack length, $a$, allows us, in principle, to ascertain $a$, and its evolution, $a(t)$, with time, $t$, from measurements of $\left|\varepsilon_{s}(x)\right|$, with a knowledge of $\Delta, h$ and a single value of $x$, and with the proviso that the value of $x$ in question is in the unbonded section of the adherend, and greater than 0 . (Young's modulus is not required, as long as it remains constant). However, it is clearly wiser to estimate crack length from several measurements of $\left|\varepsilon_{s}(x)\right|$, at different values of $x$, both in order to check the reproducibility of the procedure and to consider potential errors. With several strain gauges in place, at positions defined by $x_{i}$, this may be done using a statistical treatment. The apparent crack length, $a_{a p p}$, thus found is given by:

$$
\begin{aligned}
& a_{\text {app }}=\sqrt[3]{\frac{3}{2} h \Delta \frac{1}{|d \varepsilon / d x|}}=\sqrt[3]{\frac{3}{2} h \Delta \frac{\sum x_{i}^{2}}{\sum\left|\varepsilon_{i}\right| x_{i}}}, \\
& a_{\text {app }}(t)=\sqrt[3]{\frac{3 h \Delta}{2 \alpha(t)}},
\end{aligned}
$$

where $\alpha(t)$ is the time-dependent slope of $\varepsilon$ versus $x$. Energy release rate can be found from the standard energy balance expression:

$G+\frac{1}{b} \frac{\partial U}{\partial a}=0$

leading to:

$$
G_{E B}=\frac{3 E \Delta^{2} h^{3}}{8 a_{a p p}^{4}},
$$




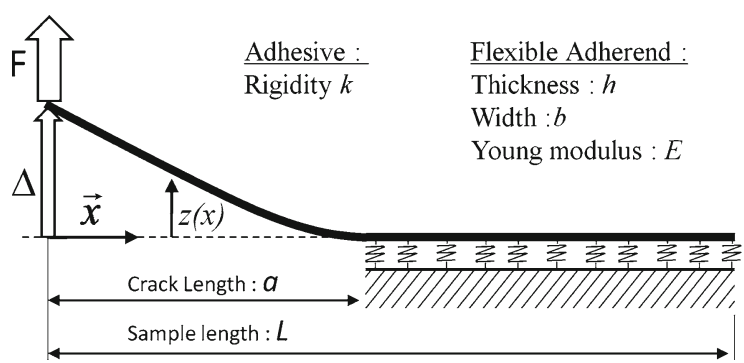

Fig. 3 Representation of adhesive joint as beam on elastic foundation (Winkler)

where the suffix EB refers to this simple derivation based on the Euler-Bernoulli model.

\subsection{Winkler (W) elastic foundation}

The rigid clamping condition of the beam at the crack tip is incorrect, strictly speaking, although it may suffice as a good approximation for a relatively rigid bondline (thin, high modulus adhesive, etc.). It does not account for compliance due to adhesive layer elasticity. When the system is loaded in mode I [in reality, a small axial component (mode II) will be present since the force introduced by the wedge acts perpendicular to the neutral axis, however this effect can be neglected (Budzik et al. 2009a)], a one parameter Winkler elastic foundation model, as shown schematically in Fig. 3, is adequate for modelling the stress redistribution near the crack tip (process zone).

The adhesive is taken as elastic (perpendicular to the bondline) and produces a tensile reaction $q(x)=$ $-k z(x)$ per unit length, proportional to and opposite to the local beam deflection $z(x)$ in the bonded zone. The stiffness $k$ is characteristic of the elastic properties of the adhesive:

$k \approx \frac{E_{A}}{e} b$,

where $E_{A}$ is adhesive Young's modulus, $e$ its thickness and $b$ the sample width.

The equilibrium relation within the bonded zone is as follows (Budzik et al. 2011a):

$\frac{d^{4} z}{d x^{4}}+\frac{k}{E I} z=0, \quad a<x<+\infty$.

The same equation, but with $k=0$, applies in the unbonded section $(0<x<a)$. (There is no reaction from the adhesive.) It is assumed that the process zone size is small compared to the sample total length to avoid any interaction with the free end of the specimen (see below).

For the free part, the deflection is given by a 3rd order polynomial:

$$
\left.z(x)\right|_{0} ^{a}=A_{2} x^{3}+B_{2} x^{2}+C_{2} x+D_{2} \quad 0<x<a,
$$

while in the bondline, the deflection decreases exponentially with a sinusoidal period:

$$
\begin{aligned}
\left.z(x)\right|_{a} ^{+\infty}= & e^{\lambda(a-x)}\left[A_{1} \cos \lambda(a-x)\right. \\
& \left.+B_{1} \sin \lambda(a-x)\right] \quad a<x<\infty .
\end{aligned}
$$

The characteristic dimension of the process zone in the following is directly related to the coefficient $\lambda$ (characteristic wave number) equal to:

$\lambda=\frac{\sqrt{2}}{2}\left(\frac{k}{E I}\right)^{1 / 4}$,

which, in turn, depends on the ratio between the tensile rigidity of the elastic layer and the bending rigidity of the flexible adherend. An important consequence is that the process zone size depends on the geometry of the test sample. We should note that the reciprocal of the wave number, $\lambda^{-1}$, expressed in units of distance, is part of the process zone, where significant adhesive strain in the opening direction occurs, and which may be referred to as the high strain zone.

The coefficients $A_{1}, B_{1}, A_{2}, B_{2}, C_{2}$, and $D_{2}$ are found which satisfy the continuity conditions of functions (9) and (10) at the crack tip location $(x=a)$ up to the third order derivative, as well as the boundary conditions at the wedge position $[z(0)=\Delta$, and $\left.d^{2} z / d x^{2}=0\right]$ and are detailed in (Budzik et al. 2011a).

From these formulae are derived the important relations necessary to analyse instrumented wedge test measurements. Firstly, we have the macroscopic data corresponding to the real energy release rate, $G$, as predicted by the Winkler elastic foundation model:

$G=4 c^{4} \frac{(1+c)^{2}}{\left(3+6 c+6 c^{2}+2 c^{3}\right)^{2}} G_{E B}$.

Secondly, the apparent crack tip length, $a_{a p p}$, as estimated from the slope of the linear strain variation in the free zone is given by:

$a_{\text {app }}=a \sqrt[3]{\frac{3+6 c+6^{2}+2 c^{3}}{2 c^{3}}}$.

In these expressions, the coefficient $c=a \lambda$, is an adimensional coefficient representing the product of 
the geometrical (real, intrinsic) crack length $a$, and the parameter indicating the process zone size, $\lambda$. For high values of $\lambda$ (equivalently small process zone), the classical fracture mechanics simple beam theory is sufficiently accurate. In crack propagation experiments, the important point is that the conventional root rotation correction methods are approximate since $c$ varies with $a$, and therefore so does the correction coefficient! Surprisingly, when estimating the energy release rate from simple beam theory with the apparent crack length, the errors compensate approximately (Budzik et al. 2011a). Although there is some doubt about whether the adhesive (polymeric) properties remain stable under applied load, this self-compensation can be used to find fracture parameters with much simpler Euler-Bernoulli approach.

Yet, we wish to take advantage of the complementary information given by strain measurement to obtain information about the process zone. To do this, strain gauges are also used to monitor strain evolution in the bonded area (where strain would be zero, assuming EB theory).

From relations (9) to (11) and appropriate constants, the theoretical strain evolution along the sample in the free part can be found:

$$
\left|\varepsilon_{S}(x)\right|=\frac{3 h \Delta}{a^{3}} \frac{c^{3}}{\left(3+6 c+6 c^{2}+2 c^{3}\right)} x \quad 0<x<a .
$$

In the bonded zone the strain evolution is given by:

$$
\begin{aligned}
& \left|\varepsilon_{S}(x)\right|=\frac{h}{2 R}=\frac{h}{2}\left|\frac{d^{2} z}{d x^{2}}\right| \\
& =\frac{3 h \Delta}{a^{2}} \frac{c^{2}}{\left(3+6 c+6 c^{2}+2 c^{3}\right)} e^{\lambda(a-x)} \\
& \quad \times(-(1+c) \sin \lambda(a-x)+c \cos \lambda(a-x)),
\end{aligned}
$$

Let us consider Eq. (15) in more detail. The bending moment increases linearly from the wedge towards the bonded area, and thus maximal strain may be expected at the crack front, just before the resistance of the adhesive layer partially offsets effects of bending (totally with the assumptions of the EB model!). The value of $x$ for maximal strain within the Winkler model is simply found by differentiation of Eq. (15) and setting the result equal to zero. This leads to:

$(2 c+1) \sin \lambda(a-x)+\cos \lambda(a-x)=0$.

With the (reasonable) simplification that $c \gg 1$, Eq. (16) suggests that maximal strain is to be found at $a=x$, the fact that it is indeed a maximum verified by the second derivative being negative. However, to be more precise, although Eq. (16) has no exact solution, it is readily shown that:

$\tan \lambda(a-x)=\frac{-1}{(2 c+1)} \approx \lambda(a-x)$,

equivalent to:

$x \approx a+\frac{1}{\lambda(2 c+1)}$.

Thus interestingly, and counter intuitively, the maximal strain, and therefore curvature, are not exactly at the crack front, but at a very small distance within the intact bond. In fact, relative to the crack length, $a$, the distance from the crack front is $1 /[c(2 c+1)]$. The distance is very small and so we may take maximal strain to be at $x \approx a$. At the other extreme, since, in general, $c \gg 1$, it can be seen that surface strain becomes zero at a value of $x$, or more specifically, $(a-x)$, corresponding to $\sin \lambda(a-x) \approx \cos \lambda(a-x)$, leading to:

$\lambda=\frac{\pi}{4\left(a-x_{0}\right)}$,

where $x_{0}$ is the required value. Use can be made of these formulae to estimate $\lambda$. Consider a given strain gauge, at a given (known) distance, $x_{i}$, from the origin, during crack propagation. We assume that the value of $x_{i}$ is sufficiently great for this position to be well within the bonded zone. When the crack has propagated to such an extent that $x_{i}=x_{0}$ (still within the bonded joint), local surface strain will be zero. If the position of the crack front, $x=a$, is known at this moment [from strain gauge measurements in the unbonded zone and use of Eq. (4), or alternatively more precisely with the Winkler equivalent], then $\lambda$ can be calculated from Eq. (18). This is shown schematically in Fig. 4 for the crack moving from position $a_{1}$ to $a_{2}$.

Having developed the required theoretical background we now present experimental findings.

\section{Results}

\subsection{Fracture}

In the basic EB treatment, crack growth is described by one variable - the strain slope $\alpha(t)$. In Fig. 5, $\alpha$ is shown for different times. The linear relation between 


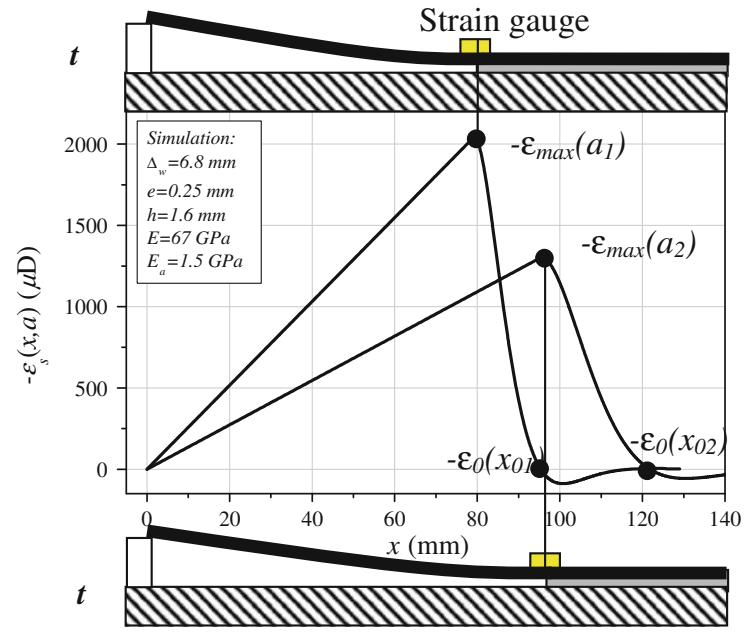

Fig. 4 Physical meaning of the maximum $\left(\varepsilon_{\max }\right)$ and zero strain $\left(\varepsilon_{0}\right)$ points for different crack positions

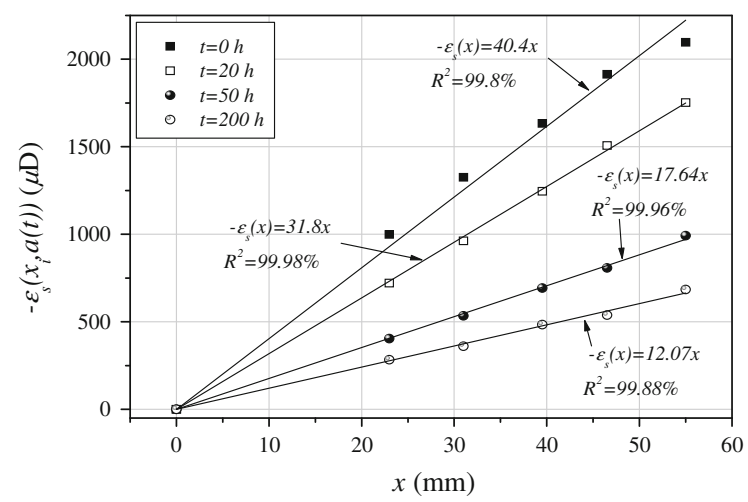

Fig. 5 Change of the strain slope with time

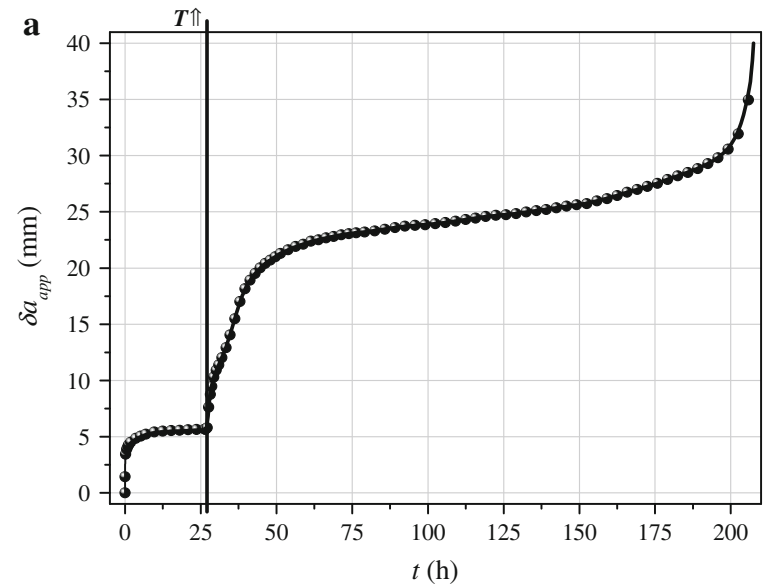

strain, $-\varepsilon_{s}$, and strain gauge position, $x$, as given by Eq. (4) is clear.

Change of the strain slope with time is directly converted to crack length, $a_{a p p} v i z$. Equation (4), from which crack growth, $\delta a$, kinetics can be deduced. In Fig. 6a, crack growth with time is shown. In Fig. 6b, crack speed $d a / d t$ is given as a function of time $t$.

Up to $c a .25 \mathrm{~h}$, crack growth seems fairly classic, with rate decreasing due to reduced $G\left(\sim a^{-4}\right)$, the final value of $d a / d t$ being ca. $0.01 \mathrm{~mm} / \mathrm{h}$. At this stage, the temperature was increased from 27 to $40^{\circ} \mathrm{C}$ (bold, vertical lines in Fig. 6). Crack acceleration is clear. Since strain energy release rate, $G$, remains constant (substrate elasticity and geometry being effectively unchanged over the small period needed to change temperature), whereas fracture resistance of the polymeric adhesive generally decreases with increasing temperature, thus more rapid crack growth may be expected. This is corroborated by the DMTA results presented in Fig. 2, where it can be seen that a significant drop in modulus occurs near $40^{\circ} \mathrm{C}$. Following this, once the new, higher temperature, regime has been stabilised, crack growth decelerates, again due to decreasing $G$. (The apparent increase, starting from $c a .125 \mathrm{~h}$, is discussed below.)

Using Eq. (6), energy release rate/fracture energy can be presented as a function of crack speed (Fig. 7). This representation emphasises results shown in Fig. 6. A rapid increase of $d a / d t$ (note logarithmic scale) is seen at constant $G$ (at $c a .110 \mathrm{Jm}^{-2}$ ), corresponding to the temperature increase. Before and hereafter,

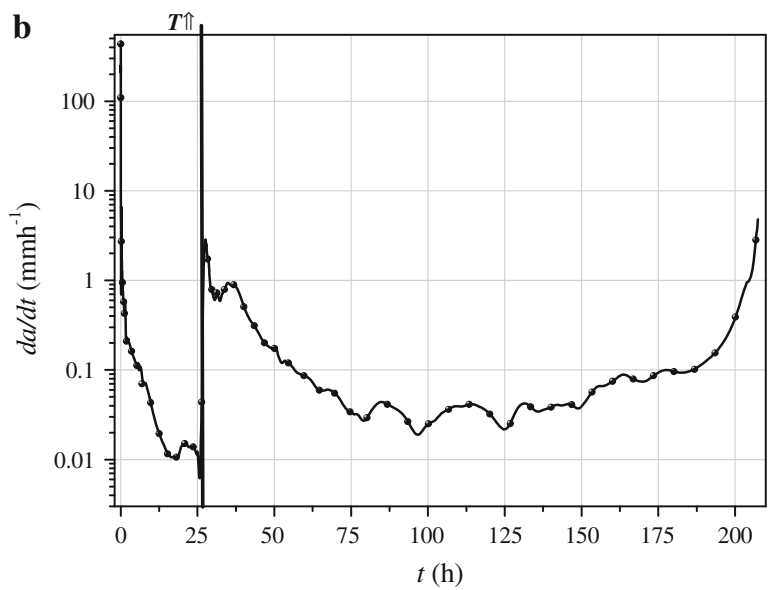

Fig. 6 Crack growth kinetics. a Crack growth, $\delta a$, versus time, $t$. b growth rate, $d a / d t$, versus $t$. Vertical lines at $c a$. $25 \mathrm{~h}$ represent temperature change 


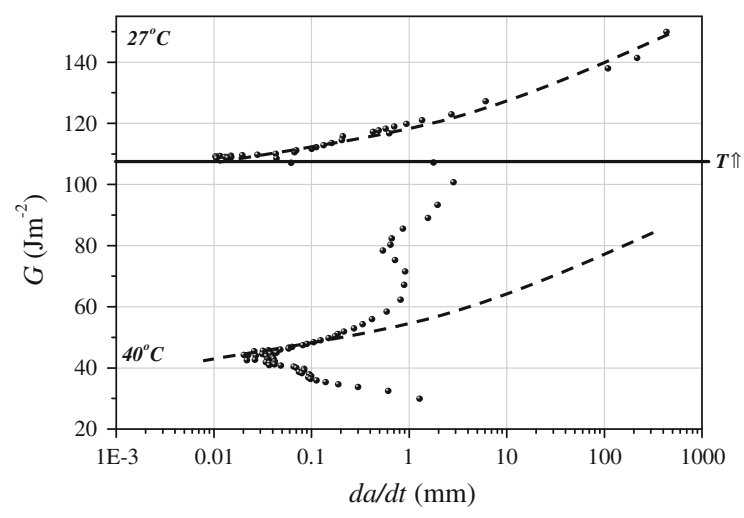

Fig. 7 Energy release rate, $G$, versus crack speed, $d a / d t$

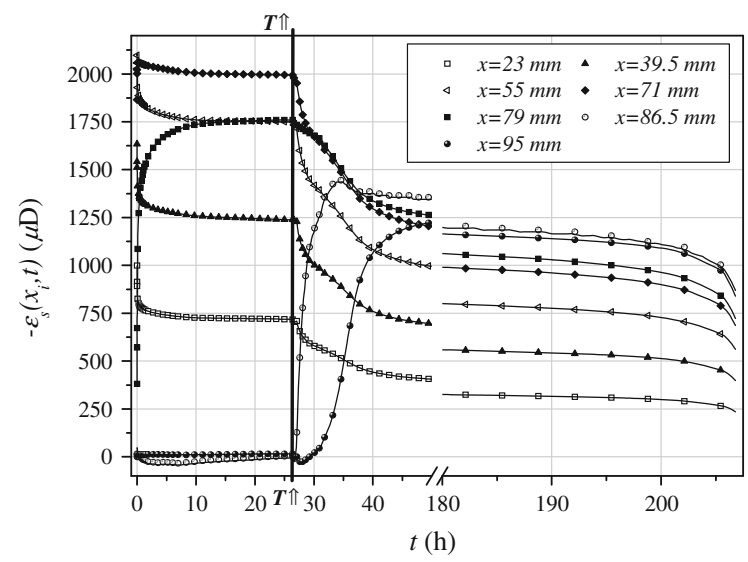

Fig. 8 Strain in time evolutions as recorded by the strain gauges bonded at different distances from the wedge, $x_{i}$

$d a / d t$ decreases concomitantly with $G$, apart from a 'hump' towards $80 \mathrm{Jm}^{-2}$. This type of continuous monitoring test is capable of picking up such local behaviour, possibly due to bond imperfections. More interestingly, the crack accelerates below a value of $G$ of $c a .40 \mathrm{Jm}^{-2}$, with decreasing $G$ ! this effect will be discussed below.

\subsection{Evolution of strain}

Results described to present have been limited to treatment of global fracture properties of the joint, obtained from data pertaining to the unbonded part. However, strain evolution can be followed with the instrumented wedge. In Fig. 8, strain, $-\varepsilon_{s}$, is plotted against time, $t$. Note that in order to keep the presentation clear the number of gauges shown is limited.

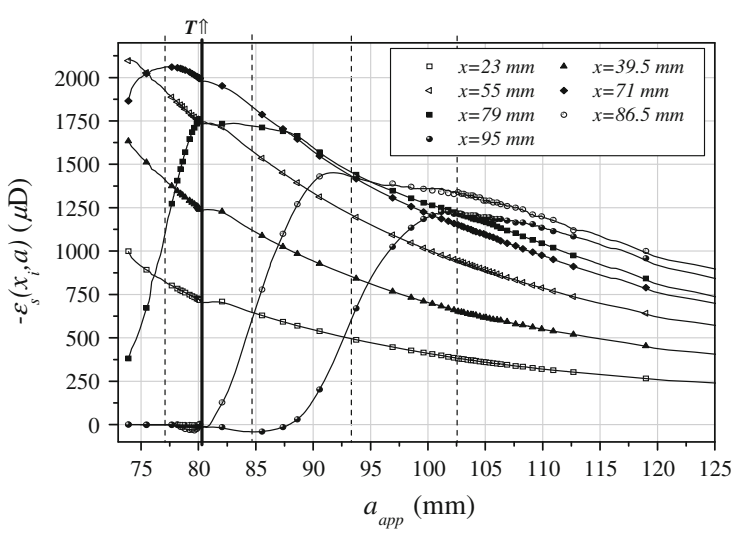

Fig. 9 Strain, $-\varepsilon_{s}$, versus apparent crack length, $a_{a p p}$. The dashed line indicating defined gauge positions does not correspond to the value of the apparent crack length

As crack length increases, gauges initially corresponding to the bonded joint, starting from the gauge at $x=71 \mathrm{~mm}$, traverse a maximal value of (absolute) strain as the crack front passes near, or 'over'. Temperature increase is manifested by drop of the strain after $c a .25 \mathrm{~h}$ (indicated with a bold line). Also final acceleration is clearly visible. For further analysis and to take full advantage of the gauges initially placed in the bonded zone, it is convenient to the replace time scale by the apparent crack length, $a_{a p p}$, as obtained from EB treatment. Also, the change in coordinates renders the strain signal evolution independent of crack propagation kinetics. In Fig. 9, strain is plotted as function of apparent crack position, from which various observations may be made. Change of temperature is now represented by a small signal perturbation, but more intriguing, by the extension of the signal peak region and longer process zone (loaded zone in front of the gauge). Accordingly, signal peaks, expected when the crack tip is exactly at the gauge position, (dashed line in Fig. 9) do not match the apparent crack position.

\section{Analysis and discussion}

\subsection{Temperature impact}

Consider an adhesive joint subjected to a temperature increase and loaded sufficiently lightly for fracture not to occur (immediately). Strain along the flexible beam is recorded by the strain gauges bonded (virtually 


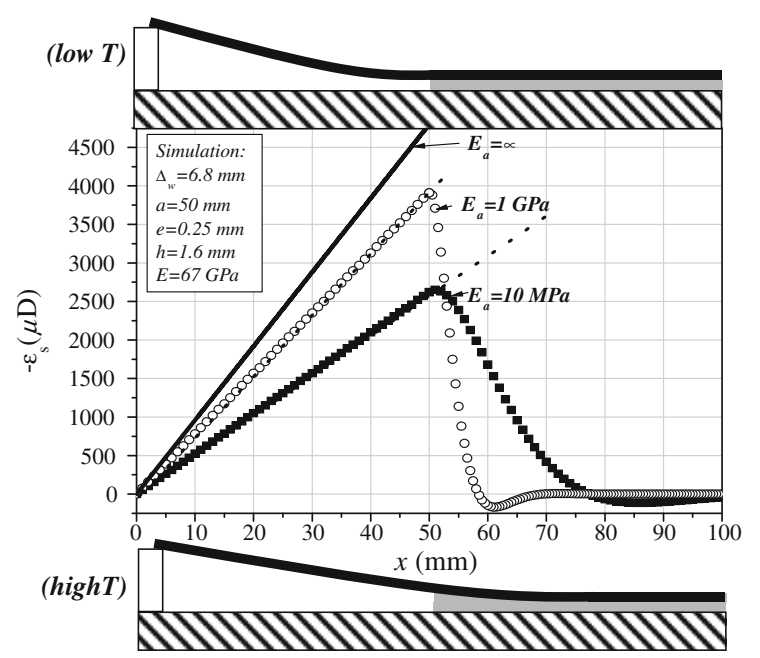

Fig. 10 Effect of change of adhesive modulus or equivalently temperature on measured surface strain. Bold line corresponds to the EB solution for actual crack position while points and squares to Winkler model solutions. Accordingly, dashed lines corresponds to the EB solutions and apparent crack positions

continuously) on both, unbonded and bonded parts of the beam like shown schematically in Fig. 10.

Increased temperature leads to lower adhesive modulus, as observed in DMTA experiments. Applying EB analysis, this will be manifested by apparent crack length growth, without real fracture! Strain recordings decrease (in absolute value), since the more supple adhesive allows higher root rotation (at the crack tip), thus decreasing the strain/distance slope: the only variable available in the EB model (see Fig. 10 where dashed lines corresponds to EB strain distribution). If the real crack length is not yet known, and limiting gauge readings to those in the free zone, the Winkler treatment cannot be used without knowledge of adhesive properties. Strain recorded in free zone is necessarily the same for both EB and Winkler models. However, strain gauges beyond the crack front, in the bonded zone, supply information about the process zone. Data from both zones of the joint allow the strain peak at the crack front to be isolated (maximum of bending moment, as observed for 'passed over' gauges in Fig. 9). When the temperature increases, the strain distribution changes. However, applying the Winkler model to strain values gathered from the entire sample length leads to the same intrinsic crack position, but with reduced adhesive properties to follow the corrected strain trend (see Fig. 10). There is, however, a simpler procedure, described below.

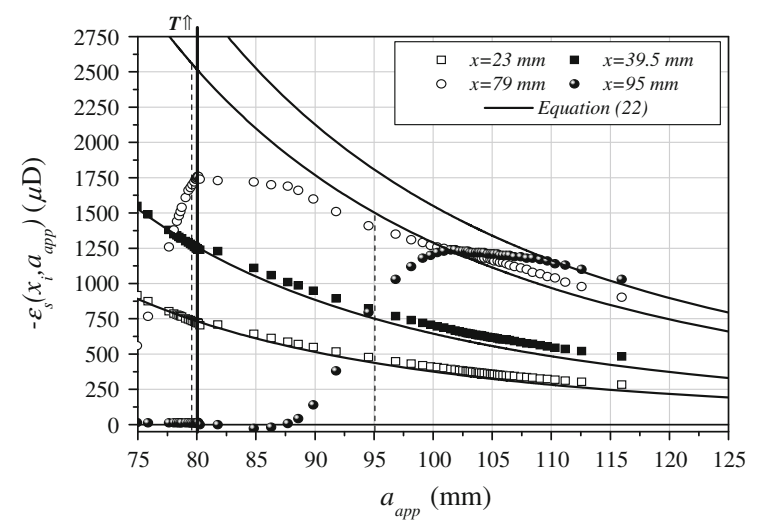

Fig. 11 Strain versus apparent crack position together with theoretical evolution

\subsection{Theoretical and experimental strain distribution: crack length correction}

It has been ascertained that the EB treatment leads to an incorrect estimate of crack length, $a_{a p p}$. This has little bearing on estimated fracture energy $(d a / d t$ is only slightly affected). However if, for example, environmental effects are of interest, it is of major concern to find the real crack length, $a$. In principle, this can be found using the Winkler model [Eq. (13)], but this requires yet unknown properties of the adhesive.

From EB theory, the theoretical strain distribution for gauges in the unbonded section is accessible $(x \leq a)$. Strain evolution of the gauge placed at $x$ depending on crack position $a$ is given by Eq. (3).

In Fig. 11, strain data are plotted together with the theoretical trend. At the lower of temperature, the theoretical curve fits experimental data very well, while at increased temperature, some disparity is seen although acceptable and still validating the elastic approximation. More importantly, the dashed line, indicating apparent position, does not correspond to the maximum of the strain signal as expected $\left(\varepsilon_{s} \approx F x\right)$. Clearly, apparent crack length is not the actual value and requires appropriate correction. This can be done using Eq. (13), although it is not straightforward, due to the cubic form. In Eq. (13), $c$ is a function of temperature [cf. Equations (7) and (11)]. This is actually visible since the temperature increased at about the time that the crack was passing over the gauge at $x=79 \mathrm{~mm}$. This reveals a change in process zone size: the 'hump' is more spread out at the higher temperature, while sharp 
for the lower temperature. It may be assumed that the strain maximum is incorrect for the gauge at $95 \mathrm{~mm}$.

Knowing the position of strain gauges in the bonded zone, we can find the correct crack length. This can be done by direct comparison between the apparent position of the maximal strain signal (and strain distribution) and the strain recorded by the gauge at a known position. This leads to the simple expression:

$a=\frac{x}{a_{a p p}(x)} a_{a p p}$,

where $x$ is gauge position and $a_{a p p}(x)$ is a corresponding apparent crack length at $x$.

From Fig. 11, for the bond extremity, we find that the apparent crack length leads to a value of $125 \mathrm{~mm}$ when the entire plate is of only of length $120 \mathrm{~mm}$ ! The contact line between wedge and plate was also not exactly at the plate tip and moreover we inserted PTFE strips to define the bonded zone. These facts together lead to maximum possible crack length of $c a .110 \mathrm{~mm}$ (and so at this stage, the apparent length is greater than possible by $15 \mathrm{~mm}$ !). Comparing real and apparent values, we found that the correction $x / a_{a p p}(x)$ is not constant and the empirical relationship found was:

$a=A \ln \left(a_{\text {app }}\right)-B$,

where $A=81.7, B=284.1$ (with a coefficient of determination $\left.R^{2}=99.9 \%\right)$. This interesting finding shows that $\lambda$ changes during the test, $\left(a / a_{a p p}\right.$ is not constant). In Fig. 12, strain evolution versus corrected crack length is plotted.

At this stage, it is good to appreciate some of the experimental facts. Using the EB model, the strain versus crack position curve will not show any change

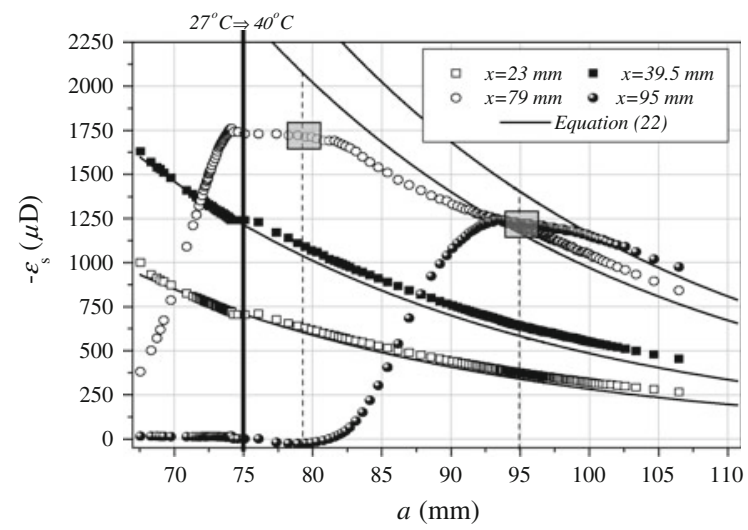

Fig. 12 Strain $-\varepsilon_{s}$ evolution as a function of intrinsic crack length $a$ of the signal up to the gauge position $\left[\varepsilon_{s}(a<x<\infty)\right]$ indicated by the dashed lines. After this point, evolution will follow Eq. (3) which is shown with a bold continuous line. The experimental curves do not follow this rule. First of all, due to the adhesive elasticity (and possibly other effects), the strain increases when the crack is close to the gauge. Thus, no sharp transition between free and bonded parts is observed. Several explanations are possible. The theoretical curve is limited to the elastic case while some adhesive plasticity can be expected (Tvergaard and Hutchinson 1993, 1994, 1996). Moreover, the test is conducted (partially) at a temperature close to $T_{g}$, thus time-dependent flow and relaxation are possible. The sheer physical size of strain gauge grid is of about $2 \mathrm{~mm}$ (represented schematically by the grey rectangle in Fig. 12), so that the signal recorded is not explicitly the actual value of strain at $x$, being averaged over the length (although this effect is very small compared to the others). Notwithstanding, this results leads, to our knowledge, to the most precise information about joint fracture and bulk properties yet given.

\subsection{Properties of the adhesive}

Analysis to present has been global. The method and analysis proposed could be used to follow the evolution of bulk adhesive properties. This could be particularly important for following such things as environmental degradation of the adhesive. Application of Eqs. (14) and (15) is made to experimental data to get the best fit, by minimising overall errors (regression). This procedure requires finding two, in the present conditions non stationary, variables $\lambda$ and $a$. Successful application has been made in stationary, non-destructive applications (Budzik et al. 2011c), and can be applied virtually at any stage of the test. Results of fitting of the theoretical curve [Eqs. (14) and (15)] to the experimental strains is shown in Fig. 13a.

Notwithstanding, the present method can be used to evaluate bulk adhesive properties. Directly from the strain measurements having corrected, intrinsic, crack position now and defined gauge position we can use the fact that the characteristic wave length $\lambda^{-1}$ (assuming that the process zone is small comparing to crack length) can be given simply by:

$\lambda^{-1}=\frac{4\left(a-x_{0}\right)}{\pi}$,

with $x_{0}$ being position of the 0 strain as recorded by the gauge passed by the crack front at $a=x$. 

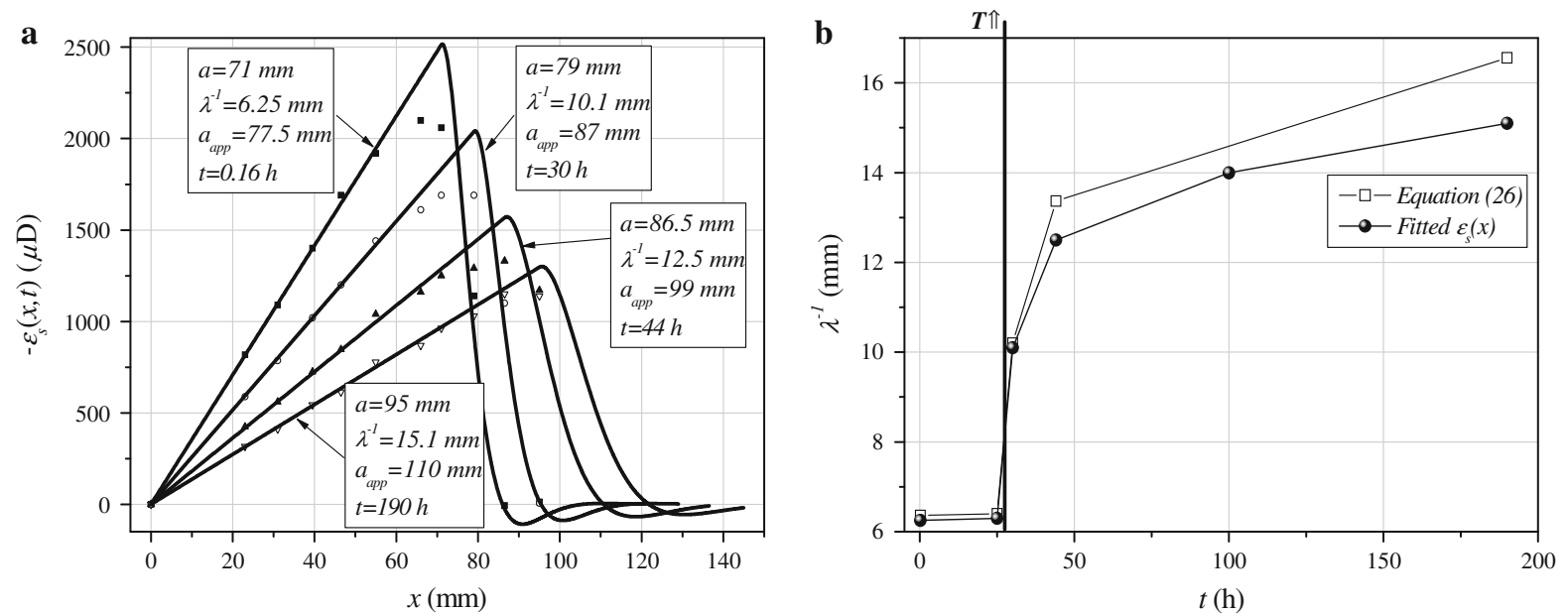

Fig. 13 a Experimental strain distribution $-\varepsilon_{S}(x)$ results together with theoretical Winkler functions for different time of test. b Characteristic wave length $\lambda^{-1}$ evolution in time, as obtained with both estimation methods

A disadvantage of this method is the need to wait until the crack front 'passes over' the gauge position.

In Fig. 13a, surface strain for different times and different temperatures are shown. The two methods used, viz. fitting Eqs. (14) and (15), and applying Eq. (21), give good agreement. In Fig. 13b the size of the process zone, as obtained from both methods is plotted as a function of crack propagation time. An additional point is given for fitted $\lambda^{-1}$ at $t=100 \mathrm{~h}$ to follow more precisely the trend of the process zone growth. As stated above, this fitting procedure can be applied virtually continuously. Accordingly, at the lower temperature $(t<25 \mathrm{~h}) \lambda^{-1}$ is evaluated at about 5.6-6 mm. DMTA results show a rapid change of the adhesive properties in the temperature range of interest. This is observed at between $25-30 \mathrm{~h}$, when $\lambda^{-1}$ rapidly increased from 6.2 to $10 \mathrm{~mm}(N . B$. this corresponds to a $c a .10 \times$ change of the adhesive Young's modulus, which is consistent with DMTA). Estimations were, in fact, not made continuously, and probably this increase (estimations made with $5 \mathrm{~h}$ intervals) was indeed during the time of temperature increase only. More importantly, this effect is visible with both methods, regardless of the propagating crack front! After $c a .30 \mathrm{~h}$, when the gauge at $x=79 \mathrm{~mm}$ is passed (and new conditions are set), $\lambda^{-1}$ varies following a logarithmic trend. There are two possible scenarios, first: the adhesive may undergo further modifications in its structure, leading to some degradation, or more probably, the adhesive undergoes viscous flow. Of course, such situations are not considered in our purely elastic analysis. However we have showed that $\lambda^{-1}$ and advanced analysis of this parameter can be used to provide good crack length estimations, and potentially lead to further developments, an example of which is the 'apparent' $\lambda$ concept (Chauffaille et al. 2011).

\subsection{Finite length effect}

In the classic asymmetric wedge test, we generally assume that the flexible beam is long enough so that the process zone is small compared to the sample size. In the present experiment, the calculated crack front position 'exceeds' the limit of the bonded zone at the very end of the experiment (see Fig. 11) so that the process zone, in advance of the crack front, may cover the entire length of the remaining bonded zone! This problem has already been observed but not recognised (Karac et al. 2011). Indeed, the speed versus time plot (Fig. 6b) reveals clear crack acceleration starting from the middle of the test, $c a .100 \mathrm{~h}$, which continued up to the end. To evaluate this effect, the elastic foundation model must be extended to take into account the finite length of the sample. In this case, the beam deflection is given by the relations:

$$
\begin{aligned}
& \left.z(x)\right|_{0} ^{a}=A^{\prime} x^{3}+B^{\prime} x^{2}+C^{\prime} x+D \\
& \left.z(x)\right|_{a} ^{+\infty}=e^{\lambda(a-x)}\left[A^{\prime \prime} \cos \lambda(a-x)\right. \\
& \left.\quad+B^{\prime \prime} \sin \lambda(a-x)\right]+e^{-\lambda(a-x)} \\
& \quad \times\left[C^{\prime \prime} \cos \lambda(a-x)+D^{\prime \prime} \sin \lambda(a-x)\right] .
\end{aligned}
$$




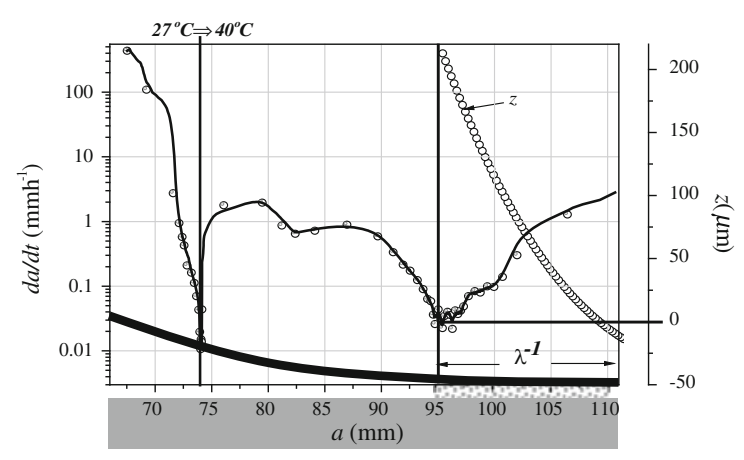

Fig. 14 Crack speed versus crack position

Expressions for the coefficients from $A^{\prime}$ to $D^{\prime \prime}$ are too complicated to be given here. Two additional terms which enables us to take into account the free edge condition of the flexible beam at $x=L$ are:

$$
\begin{aligned}
& \frac{d^{2} z}{d x^{2}}=0, \\
& \frac{d^{3} z}{d x^{3}}=0 .
\end{aligned}
$$

The situation now becomes the case when the process zone is approaching the far end of the plate, giving rise to deflection in that place and consequently increasing cleavage stress acting on the adhesive. In Fig. 14, crack speed $d a / d t$ is plotted versus crack position.

The acceleration starts about $15 \mathrm{~mm}$ from the bond far end, which is about the length of the characteristic wave $\lambda^{-1}$. Therefore, a deflection axis is added to show what is the corresponding cleavage stress state

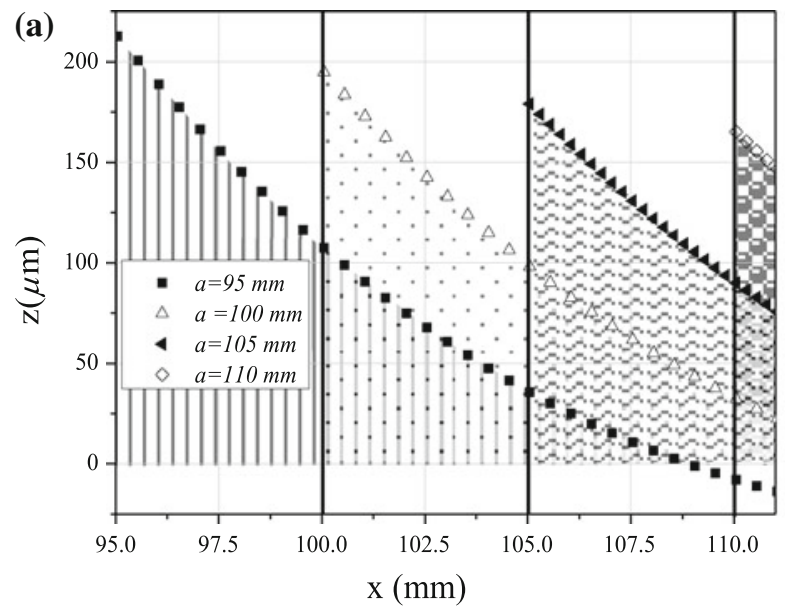

(assuming linear elasticity $z \sim \sigma$ ). At $a=95 \mathrm{~mm}$ we have maximal adhesive deflection and thus maximal cleavage stress is expected at the crack front. However, at the sample edge $(\mathrm{ca} .110 \mathrm{~mm})$ there is already some stress. Naturally, the closer the crack gets to the end, the smaller is the remaining bonded surface, and thus the higher is the average stress acting on it. Consequently, this leads to the crack acceleration. The scenario is schematically shown in Fig. 15, where in (a), deflection distribution for the new crack positions is plotted with the filled area indicating the remaining bonded zone under cleavage load. In Fig. 15b, end displacement (stress) is plotted as a function of actual crack position.

\section{Conclusions}

The wedge test is an easy and useful technique to study the impact of environment and/or technological processes on the fracture properties of adhesive joints. However it often tends to be used as a qualitative rather than quantitative tool. In this contribution, an asymmetric bonded joint, made from two aluminium alloy plates bonded with a commercial epoxy resin adhesive, was analysed using the newly developed instrumented wedge test procedure, in which strain gauges are bonded along, and on the outside of, the joint, effectively converting the bonded flexible substrate into a specific load cell. In addition, during the test, the joint was submitted to a change (increase) in temperature. This causes modifications of the adhesive mechanical properties, which can now be treated quantitatively

Fig. 15 a Deflection in the bonded part for different crack positions. b End deflection as a function of actual crack position

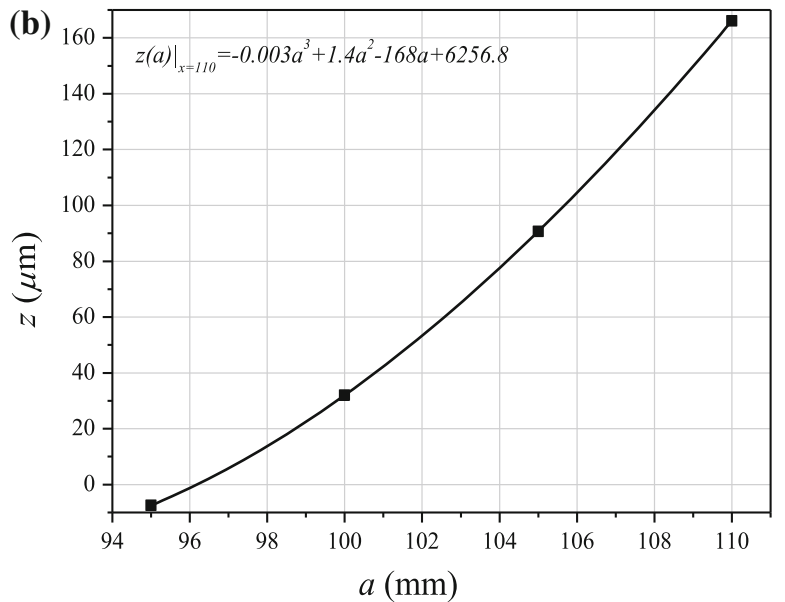


by the wedge test. In situ measurement of adhesive properties is not easy, but the present analysis sheds considerable light on possibilities. By studying strain gauge recordings, we have obtained information, not only about the global fracture properties of the joint, $v i z$. crack growth increment, crack speed and fracture energy, but also about bulk properties of the adhesive used to form the joint. The method introduced gives direct information about the process zone created in front of the crack, in which the adhesive is under significant strain and which can be followed during the test allowing estimates of the adhesive state to be made. Moreover, using the process zone characteristic parameter we have not only obtained bulk adhesive properties but also successfully analysed and explained finite specimen length effects, which lead to crack acceleration at the end of mode I fracture tests. As a perspective, we conclude that the instrumented wedge test method offers potentially much more than a simple comparative technique. By using raw strain data as a function of the crack length, we are effectively getting access to local deflection and load distributions, and thus linking global fracture energy considerations with local load singularities.

Open Access This article is distributed under the terms of the Creative Commons Attribution Noncommercial License which permits any noncommercial use, distribution, and reproduction in any medium, provided the original author(s) and source are credited.

\section{References}

Adams RD, Cowap JW, Farquharson G, Margary GM, Vaughn D (2009) The relative merits of the Boeing wedge test and the double cantilever beam test for assessing the durability of adhesively bonded joints, with particular reference to the use of fracture mechanics. Int J Adhes Adhes 29:609-620

Aglan H, Abdo Z (1996) An innovative approach to fatigue disbond propagation in adhesive joints. J Adhes Sci Tech 10:183-198

Amrutharaj GS, Lam KY, Cotterell B (1995) Fracture process zone concept and delamination of composite laminates. Theor Appl Fract Mech 24:57-64

ASTM D3762-98 Standard Test Method for Adhesive-Bonded Surface Durability of Aluminum (Wedge Test)

Baker A (1999) Bonded composite repair of fatigue-cracked primary aircraft structure. Compos Struct 47:431-443

Baldan A (2004) Review adhesively-bonded joints and repairs in metallic alloys, polymers and composite materials: adhesives, adhesion theories and surface pretreatment. J Mat Sci 39: $1-49$
Bardis J, Kedward K (2001) Effects of surface preparation on long-term durability of composite adhesive bonds. US Department of Transportation Federal Aviation Administration, Final Report, DOT/FAA/AR-01/8

Blackman BRK, Kinloch AJ (2001) Fracture tests for structural adhesive joints. Eur Struct Integr Soc 28:225-267

Boller C, Ihn JB, Staszewski WJ, Speckman H (2001) Design principles and inspection techniques for long life endurance of aircraft structures. In: Proceedings of the 3rd international workshop on structural health monitoring. Stanford/CA, USA, September 12-14, 2001

Budzik MK, Jumel J, Imielinska K, Shanahan MER (2009) Accurate and continuous adhesive fracture energy determination using an instrumented wedge test. Int J Adhes Adhes 29:694-701

Budzik MK, Jumel J, Imielinska K, Shanahan MER (2009) Fracture in composite/aluminium joints of variable adhesive properties. J Adhes 85(10):736-754

Budzik MK, Jumel J, Imielinska K, Shanahan MER (2011) Effect of adhesive compliance in the assessment of soft adhesives with the wedge test. J Adhes Sci Tech 25:131-149

Budzik MK, Jumel J, Shanahan MER (2011) Adhesive compliance effect in mode I separation: profilometry approach. Int J Adhes Adhes 31: 135-145. doi:10.1016/j.ijadhadh.2010. 11.012

Budzik MK, Mascaro B, Jumel J, Castaigns M, Shanahan MER (2011c) Monitoring of crosslinking of a DGEBA-PAMAM adhesive in composite/aluminium bonded joint using mechanical and ultra-sound techniques. Int J Adhes Adhes. Submitted

Bujanda A, Copeland C, Dibelka J, Forster A, Holmes L, Jensen R, Kosik W, McKnight S, Koellhoffer S, Gillespie Jr J (2008) Analysis of adhesively bonded ceramics using an asymmetric wedge test. Army Research Laboratory Report, ARL-TR-4665

Chauffaille S, Jumel J, Shanahan MER (2011) Pre-cracking behaviour in the single centilever beam adhesion test. Int J Fract 169:133-144

Cotterell B, Hbaieb K, Williams JG, Hadavinia H, Tropsa V (2006) The root rotation in double cantilever beam and peel tests. Mech Maters 38:571-584

Crocombe AD, Ong CY, Abdel-Wahab MM (2002) Investigating fatigue damage evolution in adhesively bonded structures using backface strain measurement. J Adh 78:745776

Crosley PB, Ripling EJ (1991) A thick adherend, instrumented double-cantilever-beam specimen for measuring debonding of adhesive joints. J Test Eval 19:24-28

Hadavinia H, Kinloch AJ, Little MSG, Taylor AC (2002) The prediction of crack growth in bonded joints under cyclicfatigue loading I. Experimental studies. Int J Adhes Adhes 23(6):449-461

Hwang HY, Kim BJ, Chin WS, Kim HS, Lee DG (2005) Prediction of crack length and crack growth rate of adhesive joints by a piezoelectric method. J Adhes Sci Technol 19:10811111

Kanninen MF (1973) An augmented double cantilever beam model for studying crack propagation and arrest. Int J Fract 9(1):83-92

Karac A, Blackman BRK, Cooper V, Kinloch AJ, Rodriguez Sanchez S, Teo WS, Ivankovic A (2011) Modelling the fracture 
behaviour of adhesively-bonded joints as a function of test rate. Eng Fract Mech 78:973-989

Ma X, Butterworth JW, Clifton GC (2009) Static analysis of an infinite beam resting on a tensionless Pasternak foundation. Eur J Mech A Solid 28:697-703

Nayeb-Hashemi H, Swet D, Vaziri A (2004) New electrical potential method for measuring crack growth in nonconducive materials. Measurement 36:121-129

Pardoen T, Ferracin T, Landis CM, Delannay F (2005) Constraint effects in adhesive joint fracture. J Mech Phys Solids 53:1951-1983

Sargent JP (2005) Durability studies for aerospace applications using peel and wedge tests. Int J Adhes Adhes 25: 247-256

Sener JY, Ferracin T, Caussin L, Delannay F (2002) On the precision of the wedge-opened double cantilever beam method for measuring the debonding toughness of adhesively bonded plates. Int J Adhes Adhes 22:129-137
Shah SP (1990) Experimental methods for determining fracture process zone and fracture parameters. Eng Fract Mech 35(1-3): 3-14 Special Issue Fracture and Damage of Concrete and Rock

Tvergaard V, Hutchinson JW (1993) The influence of plasticity on mixed mode interface toughness. J Mech Phys Solids 41:1119-1135

Tvergaard V, Hutchinson JW (1994) Toughness of an interface along a thin ductile layer joining elastic solids. Philos Mag A 70:641-656

Tvergaard V, Hutchinson JW (1996) On the toughness of ductile adhesive joints. J Mech Phys Solids 44(5):789-800

Williams JG (1989) End corrections for orthotropic DCB specimens. Compos Sci Technol 35(4):367-376

Winkler E (1867) Die Lehre von der Elasticitaet und Festigkeit. Teil 1, Verlag H. Dominicus, Prague [As noted in Fryba L (1993) History of Winkler Foundation. Veh Syst Dyn Suppl 24:7-12] 